\title{
Avaliação do padrão de recuperação nutricional de criançãs desnutridas atendidas no centro de recuperação e educação nutricional.
}

\author{
M. de F. A. Vieira, G.M.B. Solymos, M. H. do N. Souza, A.A. Ferrari, H. Unegbu, A.L. Sawaya
}

Departamento de Fisiologia, Disciplina de Neurofisiologia e Fisiologia Endócrina da Universidade F ederal de São Paulo - Escola Paulista de Medicina, São Paulo, SP.

RESUMO. Estudo de acompanhamento do padrão de recuperação nutricional de crianças desnutridas atendidas no Centro de Recuperação e E ducação Nutricional (CREN), entre setembro de 1995 e novembro de 1996.

Овј etivo. Avaliar a recuperação nutricional das crianças em termos de velocidade de ganho anual de peso/idade e estatura/idade e identificar variações do processo de recuperação de acordo com o sexo e a idade.

Métodos. Foram estudadas 38 crianças, utilizando-se os valores do incremento de peso/idade e estatura/idade em unidades de desvio padrão ou escore Z, em relação à mediana de peso e estatura do padrão NCHS, pelo método da velocidade de crescimento por incremento.

Resultados. Houve uma relação significante entre ganhos de peso/idade $(P=0,012)$ e estatura/ idade $(P=0,001)$ e tempo de internação. Os préescolares tiveram uma velocidade de ganho de peso/idade significantemente maior $(0,684$ escores

\section{NTRODUÇÃO}

O conceito de centro de recuperação nutrici onal foi proposto por Bengoa em 1955. Tratava-se de um centro organizado como um internato, ou semiinternato, cujo objetivo era o de educar as mães através da recuperação nutricional de pré-escolares e lactentes com desnutrição primária ${ }^{1,2}$.

No Brasil, há registro de uma experiência com centros de recuperação nutricional em Pernambuco, nos anos de 68-693. Tal projeto, contudo, não teve continuidade.

E m 1994 iniciou-se em São Paulo um Centro de Recuperação e Educação Nutricional (CREN) a partir do trabalho realizado em favelas por profissionais da área de saúde e nutrição da Universidade Federal de São Paulo/ Escola Paulista de Medicina.

A recolocação da proposta de criação de centros de recuperação ocorreu por dois motivos. Primei ro, a prevalência da desnutrição ainda é bastante
Z/ano) que os lactentes (0,299 escores Z/ano), excluídas as crianças com menos de seis meses de internação. Em relação à velocidade de ganho de estatura/idade, os lactentes apresentaram um ganho maior (0,794 escores Z/ano) em relação aos pré-escolares( 0,506 escores Z/ano), embora a diferença não tenha sido estatisticamente significante. Entre os sexos, a velocidade de ganho de peso/idade foi 0,540 e 0,524 escores Z/ano para meninos e meninas, respectivamente. Quanto à velocidade de ganho de estatura/idade as meninas apresentaram um ganho significantemente maior $(0,856$ escores $Z / a n o)$ que os meninos $(0,485$ escores Z/ano).

ConcLusÃo. O presente trabalho evidenciou que existem diferenças no padrão de velocidade de recuperação nutricional de acordo com a idade e sexo.

UNITERMOS: Recuperação nutricional. Desnutrição protéi co-energética. Crescimento. Antropometria.

alta no Brasil (31\%, $\mathrm{PNSN}^{4}$ ), quando comparada a outros países. Em São Paulo, por exemplo, um estudo realizado em favelas da região sul da cidade encontrou uma prevalência de desnutrição de cerca de $34 \%$ em crianças e $40 \%$ em adolescentes $^{5}$. Sabe-se que a população moradora em favelas na cidade de São Paulo era cerca de 2 milhões de pessoas em $1994^{5}$. E, segundo, estes centros, além do tratamento das crianças, ainda permitem uma inserção na comunidade e um trabalho educativo mais eficiente e sistemático do que outros programas presentes atualmente no Brasil, como os programas de distribuição de leite, merenda escolar, cesta básica etc, pois não alcançam os grupos mais vulneráveis, além de disporem de recursos escassos e insuficientes para produzir os impactos esperados ${ }^{6,7}$.

E nquanto os centros de recuperação nutricional descritos nas décadas de 1960-19702,8,9 tinham por finalidade básica diminuir a mortalidade infantil, oferecendo um tratamento rápido (2 a 3 meses) 
para lactentes desnutridos graves dada sua alta prevalência, atualmente, eles se tornam um fator identificador na comunidade de famílias de maior risco social, oferecendo um atendimento mais a longo prazo para a criança e para a família. A proposta de intervenção e o tempo de internação devem, pois, levar em conta a complexidade dos problemas sociais que atual mente assolam a família de baixa renda nas grandes cidades, e procurar a recuperação efetiva da desnutri ção até a normalidade dos critérios nutricionais.

O presente estudo teve dois objetivos: avaliar o padrão de recuperação nutricional de crianças pré-escol ares e lactentes desnutridas atendidas no Centro de Recuperação e Educação Nutricional (CREN), em termos de velocidade de ganho anual de peso/idade e estatura/idade, e identificar variações do processo de recuperação de acordo com o sexo e a idade.

\section{MATERIAL E MÉTODOS}

A população do presente estudo foi composta por crianças de 0 a 71 meses com diagnóstico de desnutrição primária, em processo de recuperação nutricional atendidas pelo Centro de Recuperação e Educação Nutricional (CREN), de segunda à sexta-feira, das $7 \mathrm{~h} 30$ às $17 \mathrm{~h} 30$, em regime de semiinternato, recebendo cinco refeições diárias e atendi mento clínico durante o período de setembro de 1994 a novembro de 1996. As refeições eram compostas por alimentos naturais pertencentes à dieta habitual das famílias atendi das eque podiam ser adquiridos ou encontrados pelas mães nos locais que normalmente freqüentavam; exemplo de cardápio diário: café da manhã - leite em pó com nescau e farinha láctea, pão com margarina; Ianche - suco de maracujá; al moço - arroz/feijão, carne de vaca, purê de batata, suco de Iaranja, maçã; lanche - leite em pó com abacate e farinha láctea, biscoito wafer e jantar - macarrão com molho e carne moída, batata e cenoura, abobrinha cozida e chuchu. A alimentação era portanto balanceada, contendo alimentos de alto valor protéico e calórico, sendo oferecida em quantidade superior a $100 \%$ das recomendações, e com custo aproximado de $\mathrm{R} \$ 24,00$ por criança por mês. Dados preliminares de ingestão alimentar em 14 crianças revelaram um consumo alimentar de $77 \%$ de $\mathrm{K}$ cal/dia em relação à crianças americanas eutróficas (RDA), ao passo que observou-se um consumo de $257 \%$ de proteínas em relação ao RDA.

A rotina do CREN estabel ecia ainda dois períodos de sono por dia, um de manhã e outro de tarde, e incluía uma série de atividades diárias (brincadeiras, atividades de pátio, jogos, etc.) por grupos etários, com a finalidade de estimulação psicopedagógica destas, o que reconhecidamente contribui para a recuperação nutricional ${ }^{10}$.

O critério de ingresso no CREN foi percentual de adequação de peso/idade menor ou igual a $80 \%$ do padrão do National Center for Health Statistics ${ }^{11}$, aproximadamente -1,60 desvio-padrão ${ }^{12}$.

Das 40 crianças internadas, foram estudadas 38 , sendo excluí das duas que apresentavam outras patologias responsáveis por crescimento deficitári o. No momento da úl tima avaliação, três crianças haviam recebido al ta e sete haviam abandonado o tratamento devido à mudança de residência. Estas crianças foram consideradas na avaliação, totalizando 38 crianças divididas em quatro grupos: pré-escolar do sexo masculino (PEM), pré-escolar do sexo feminino (PEF), lactentes do sexo masculino (LAM) e lactentes do sexo feminino (LAF).

As variáveis utilizadas para avaliar o estado nutricional foram peso, estatura e comprimento, idade e sexo. Os indicadores do estado nutricional utilizados foram peso/idade, estatura/idade, posteriormente comparados ao padrão do NCHS. As medidas de peso e estatura foram obtidas nos três primeiros dias úteis de cada mês, antes da primeira refeição da manhã.

Para a aferição do peso, a criança era colocada na balança, sem roupas, de maneira que seu peso fosse igualmente distribuído para cada lado do centro da balança, sendo registrado o valor mais próximo de 0,5 g, com a criança totalmente imóvel ${ }^{13}$. Para pesar as crianças com menos de $14,5 \mathrm{~kg}$ era usada uma balança KRATOS digital, com capacidade para $15 \mathrm{~kg}$, com precisão de 5 gramas. Para as crianças com mais do que $15 \mathrm{~kg}$, era usada uma balança FI LIZOLA de plataforma, com capacidade de $150 \mathrm{~kg}$ e precisão de 100 gramas.

O comprimento das crianças menores de 24 meses era medido com um infantômetro colocado sobre uma superfície plana, e a estatura das crianças maiores de 24 meses era medida com um estadi ômetro fixado na parede, registrando a medida o mais próximo de $0,1 \mathrm{~cm}$, segundo metodologia proposta por Lohman ${ }^{13}$.

A data de nascimento era obtida da certidão de nascimento apresentada no momento da internação. A idade da criança era então cal culada pela subtração da data de col eta das medidas e data de nascimento.

A avaliação do processo de recuperação das crianças foi realizada utilizando os valores do incremento das variáveis em unidades de desvio padrão ou escore $Z$, em relação à mediana de peso e estatura do padrão NCHS. O uso do escore Z 
permite comparações com outros indicadores e entre crianças de diferentes faixas etárias ${ }^{14}$. Com os valores obtidos foi calculada a velocidade de crescimento por incremento ( $\mathrm{VCl}$ ) segundo a fórmula proposta por Martell ${ }^{15}$ em relação ao tempo de internação em meses. Os valores obtidos pela fórmula da VCl foram então multiplicados por 12

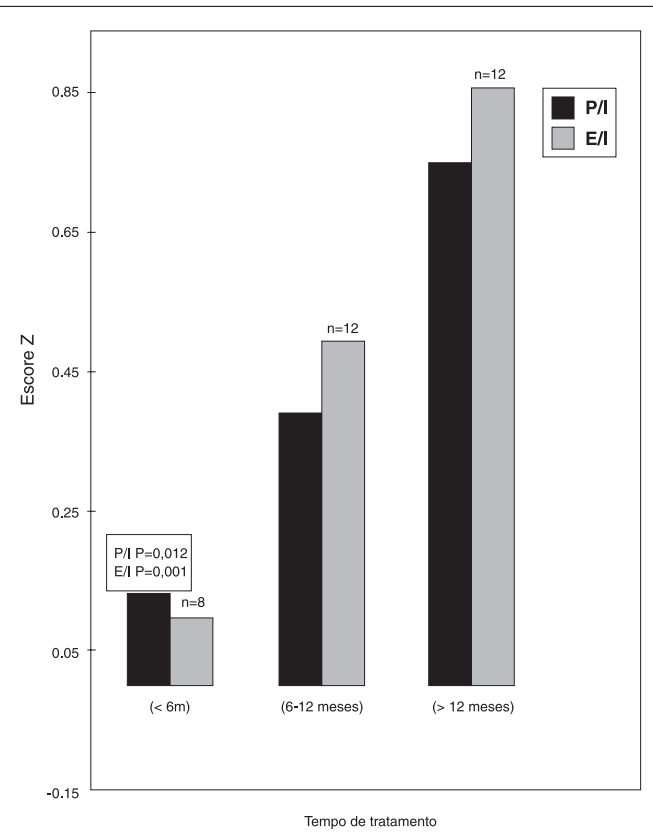

Gráfico - Média de ganho de peso/ idade $(P / I)$ e estatura/idade $(E / I)$, segundo tempo de tratamento, em escore $Z(N=38)$. para obter a velocidade de ganho de peso/idade e estatura/idade anuai s em escores Z. Para a análise estatística dos dados foram utilizados testes de anál ise de variância (ANOVA) para comparação de 3 e 4 grupos e teste t de Student para comparação entre 2 grupos (lactentes (LAC) e pré-escolares (PRE); masculino (M) e feminino (F). Os dados foram analisados para um nível de significância de $P<0,05$. F oram usados os seguintes programas na realização do trabalho: E pi info $6^{16}$ para avaliação nutricional, Systat - Systat Inc, Evanston, I.L.e SPSS for Windows para as análises estatísticas.

\section{RESULTADOS}

O tempo médio de internação das crianças estudadas foi de 11,2 meses (variando de 1 a 23,3 meses) e os ganhos médios durante esse período foram de 0,50 escores $Z$ de peso/idade $(P / I)$ e 0,58 escores $Z$ de estatura/idade (E/I ), para o conjunto das crianças ( $\mathrm{N}=38$ ).

No Gráfico ao lado, encontram-se os dados sobre ganho de peso/idade (P/I ) e estatura/idade (E/I) em valores absolutos, segundo o tempo de internação. Os grupos foram divididos em crianças com menos de 6 meses de internação $(\varangle 6 \mathrm{~m})$, entre 6 meses e 12 meses (6-12m) e com mais de 1 ano de internação ( $>12 \mathrm{~m})$. Verifica-se um aumento significante do ganho de peso/idade $(P=0,012)$ e de estatura/idade $(P=0,001)$, de acordo com o tempo de internação, sendo que o ganho em estatura é superior ao ganho em peso para crianças com mais de 6 meses de internação.

Tabela 1 - Média, Desvio Padrão, Mediana, Mínimo e Máximo de peso de nascimento em gramas (PN), idade no ingresso (meses), e escore $Z$ inicial de peso/idade (P/I) e estatura/idade (E/I), de crianças desnutridas em processo de recuperação nutricional atendidas pelo Centro de Recuperação e Educação Nutricional (CREN), segundo sexo e faixa etária ( $N=30)$.

$P N: M \pm D P$

Mediana

Mínimo

Máximo

$P / \mathrm{I}: \mathrm{M} \pm \mathrm{DP}$

Mediana

Mínimo

Máximo

$E / I: M \pm D P$

Mediana

Mínimo

Máximo

Idade:M $\pm \mathrm{DP}$

Mediana

Mínimo

Máximo

\begin{tabular}{|c|c|}
\hline \multicolumn{2}{|c|}{ Pré-Escolares } \\
\hline Masculino & Feminino \\
\hline
\end{tabular}

$2,218 \pm 1,201$

2,640

1,150

3,450

$-2,346 \pm 0,476$

$-2,410$

$-3,070$

$-1,710$

$-2,144 \pm 1,016$

$-1,875$

$-4,330$

$-0,750$

$35,275 \pm 10,874$

31,250

24,100

51,800
$2,460 \pm 0,541$

2,505

1,700

3,100

$-2,145 \pm 1,051$

$-2,570$

$-2,850$

$-0,090$

$-2,228 \pm 0,756$

$-2,350$

$-3,230$

$-1,100$

$44,050 \pm 12,646$

46,250

25,300

57,900

Masculino
$\mathbf{n}=\mathbf{7}$
$2,904 \pm 0,383$
2,940
2,300
3,460
$-2,370 \pm 0,572$
$-2,440$
$-3,170$
$-1,600$
$-1,722 \pm 1,101$
$-1,530$
$-3,380$
$-0,305$

$16,000 \pm 6,695$

19,200

5,800

22,600
P

Lactentes

Feminino

$2,600 \pm 0,137$

2,650

2,400

2,750

$-2,328 \pm 0,384$

$-2,460$

$-2,760$

$-1,730$

$-2,330 \pm 0,855$

$-2,100$

$-3,700$

$-1,640$

$18,960 \pm 3,843$

20,900

12,300

21,300 
Tabela 2 - Média, desvio padrão e mediana de velocidade de crescimento por incremento (VCl) de peso/idade (P/I) e estatura/idade (EI), em escore Z, e tempo de internação em meses (TTO) de crianças desnutridas em processo de recuperação nutricional atendidas pelo Centro de Recuperação e Educação Nutricional segundo sexo e faixa etária ( $N=30)$.

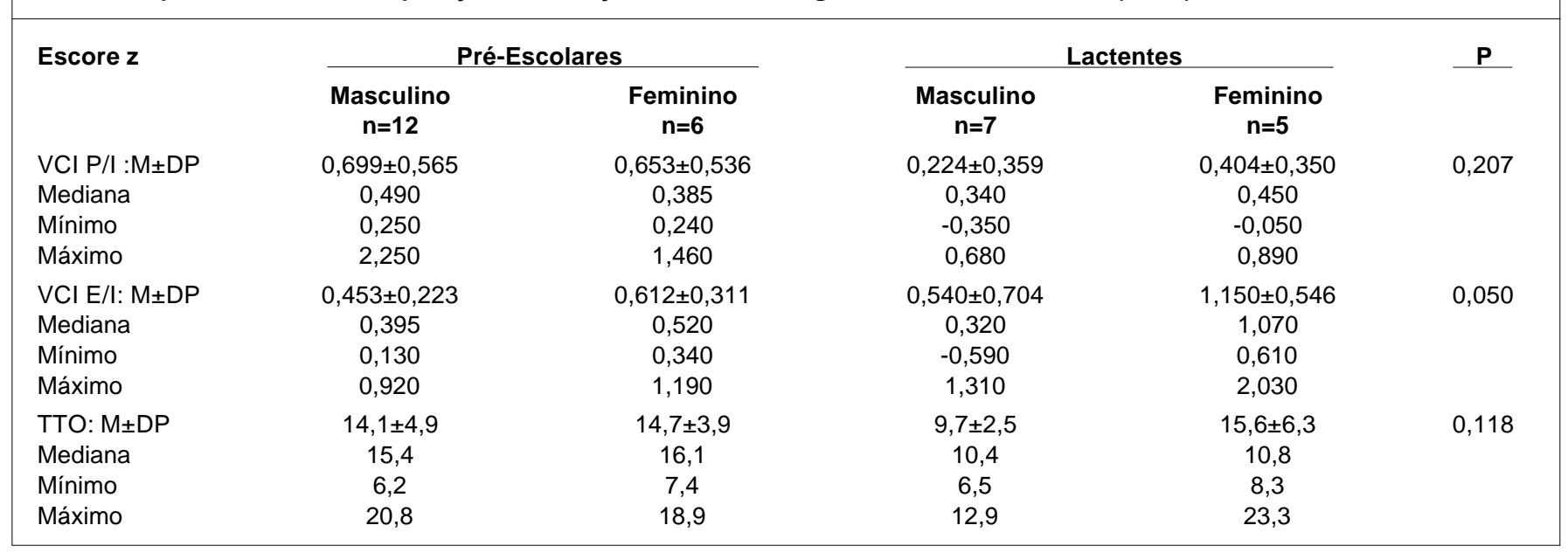

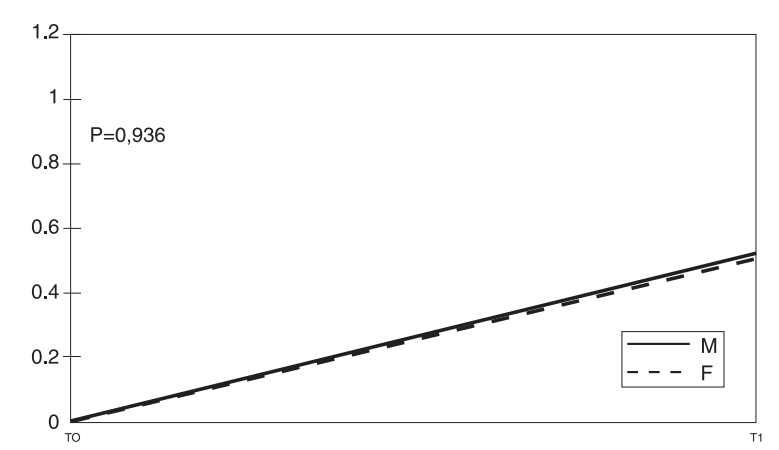

Fig. 1 - Velocidade de incremento (VCI) de peso/ idade entre as crianças do sexo masculino $(M)$ e feminino $(F)$ atendidas no CREN $(N=30)$.

Os val ores de peso de nascimento (PN) eP/I, e E/ I no ingresso das crianças desnutridas, divididas em quatro grupos de acordo com o sexo e faixa etária são observados na Tabela 1 . Considerandose o peso de nascimento, todos os grupos estudados apresentaram valores médios abaixo de $2,499 \mathrm{~g}$ (pré-escolares) e insuficente, abaixo de $2999 \mathrm{~g}$ (lactentes). A diferença entre os grupos porém não foi significante. Os valores de P/I eE/I no momento do ingresso também não apresentaram diferença estatisticamente significante.

A avaliação da recuperação nutricional pela velocidade de crescimento por incremento ( $\mathrm{VCI}$ ) foi feita considerando-se apenas as crianças com mais de 6 meses de internação $(N=30)$, divididas por faixa etária e sexo. Verificou-se uma tendência à diferenças para velocidade de ganho em estatura/idade $(P=0,050)$ mas não para ganho em peso/ idade $(P=0,207)$ entre os grupos (Tabela 2$)$.

Considerando-se o desempenho da recuperação

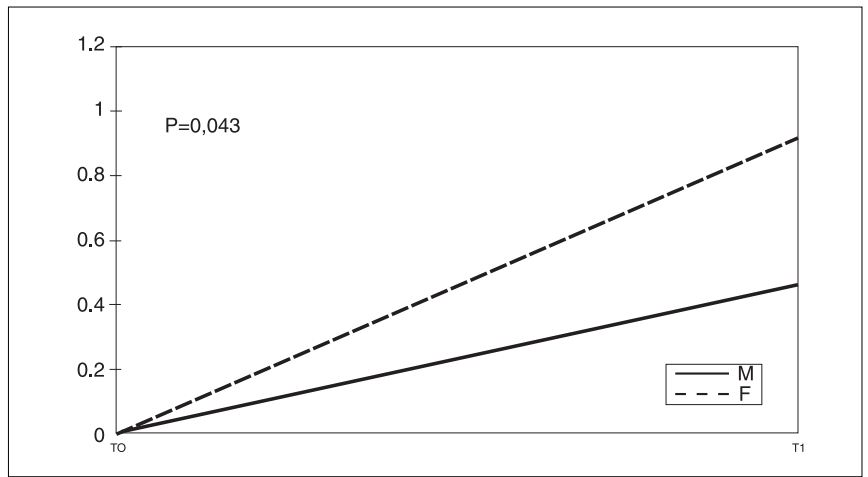

Fig. 2 - Velocidade de crescimento por incremento (VCI) de estatura/ idade entre as crianças do sexo masculino $(M)$ e feminino $(F)$ atendidas no CREN $(N=30)$.

nutricional em relação ao sexo, as meninas apresentam um padrão de recuperação para o indicador peso/idade semel hante aos meninos $(P=0,936)$ (Fig.1). Quanto ao indicador estatura/idade, elas apresentaram um incremento 1,8 vezes maior que os meninos ( $P=0,043$ ) (Fig.2).

E m relação à faixa etária, os pré-escolares apresentaram velocidade de ganho de peso/idade 2,3 vezes maior que os lactentes $(P=0,038)$ ( $F$ ig.3), enquanto que nos lactentes observou-se velocidade de ganho de estatura/idade 1,6 vezes maior do que os pré-escolares ( $P=0,116)$ (Fig.4).

\section{DISCUSSÃO}

A velocidade de crescimento tem sido proposta como método de avaliação da recuperação nutricional, visando avaliação de programas de intervenção e do desenvolvimento individual de crianças em clínicas ou ambulatórios ${ }^{17}$. É necessário, 


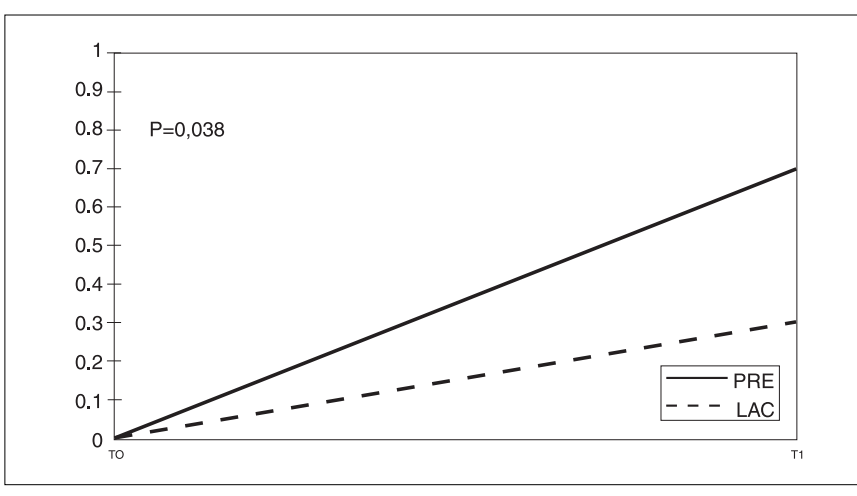

Fig. 3 - Velocidade de crescimento por incremento (VCI) de peso/ idade entre préescolares (PRE) e lactentes (LAC) atendidos pelo CREN $(N=30)$.

porém, que esta seja realizada em intervalos de pelo menos três meses, caso contrário apresenta uma variabilidade muito grande ${ }^{18}$. Tanner et al ${ }^{19}$ consideram que um período de avaliação de um ano apresenta a vantagem de eliminar variações sazonais. Segundo Lampl et a/20 o crescimento linear ocorre por saltos, e intervalos muito pequenos de observação podem levar a erros. Por esse motivo, realizou-se no presente trabal ho o cálculo da velocidade de crescimento por incremento anual apenas para as crianças com mais de seis meses de internação.

Não houve diferença estatísticamente significante entre os grupos estudados com relação aos déficits iniciais de peso/idade, estatura/idade e peso de nascimento no iníci o do tratamento. Todos grupos apresentaram média de peso de nascimento insuficiente ou abaixo de $2.500 \mathrm{~g}$, mostrando que o problema nutricional era de longa duração e provavel mente já presente durante o período gestacional.

Entre as crianças internadas no CREN, observou-se que à medida em que aumentou o tempo de internação houve um incremento tanto no indicador peso/idade como no indicador estatura/idade, com predominância da recuperação de estatura sobre o ganho de peso. Monckeberg ${ }^{9}$, ao relatar a recuperação de desnutridos no Chile, encontrou um tempo médio de internação de três meses, sendo as crianças em sua maioria menores de um ano. Massa et al. ${ }^{21}$, ao avaliarem 37 crianças peruanas atendidas em uma U nidad de Rehabilitacion Nutricional, com idades entre 2 e 28 meses sendo a maioria com desnutrição de III Grau segundo a classificação de Gomez, encontraram um tempo médio de 82 dias de internação. Comparando-se o desempenho das crianças atendidas pelo CREN, estas levam um tempo relativamente Iongo para alcançar a recuperação, o que pode ser explicado

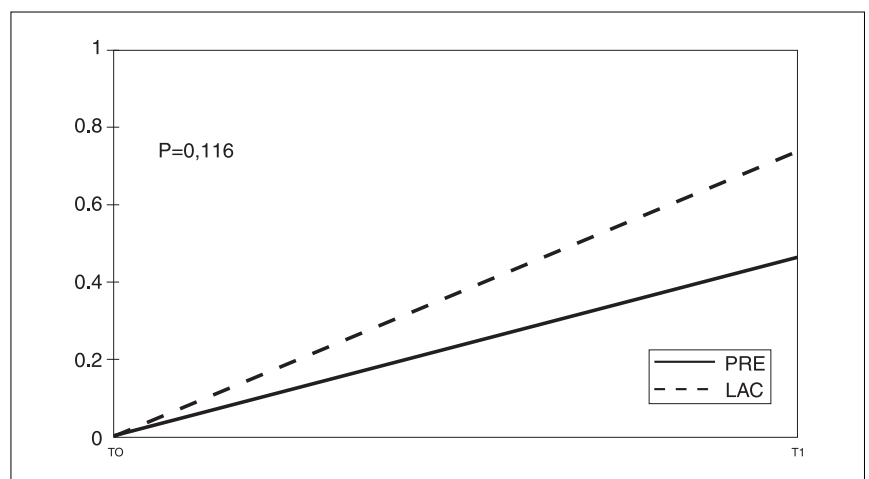

Fig. 4 - Velocidade de crescimento por incremento (VCI) de estatura/ idade entre préescolares (PRE) e lactentes (LAC) atendidos pelo CREN ( $N=30)$.

pel a idade das mesmas, a maioria com 12 meses ou mais (90\%), pelo fato de que o CREN atende em regime de semi-internato e pelo critério de alta mais exigente. O critério de alta utilizado nas experiências relatadas foi adequação de peso/estatura, facilmente alcançado quando a recuperação ocorre primeiro em peso; ao passo que para uma recuperação em estatura, como é o caso das crianças do CRE N, o tempo de recuperação é necessariamente mais longo. $\operatorname{Prader}^{22}$ propôs a utilização da doença celíaca e de Crohn como modelos para estudar a desnutrição primária. Kirschner et al..$^{23}$, estudando crianças com doença de Crohn, em tratamento com restituição nutricional oral, encontraram um aumento de estatura de $6,2 \mathrm{~cm} / a n o$ (variação de 5,5 a 8,5). Essas crianças necessitaram de 3-4 anos de intervenção nutricional para atingir o percentil no qual estavam antes de desenvolverem a doença. Barr et al. ${ }^{24}$, ao acompanharem durante três anos a recuperação nutricional de 13 crianças com doença celíaca, com idades entre 9 e 15 meses, demostraram que o tempo necessário para a recuperação completa do peso em crianças com doença celíaca foi de um ano, enquanto para a estatura foram necessários dois anos para a completa normal ização. Estes val ores são muito semeIhantes aos encontrados nas crianças atendidas pel oCREN, visto que elas levam cerca de dois anos ou mais para alcançar a normalização em peso/ idade e estatura/i dade, sendo necessário um tempo maior para os pré-escolares do que para os lactentes.

No que se refere à recuperação da estatura/ idade, observou-se um desempenho melhor entre as meninas e os lactentes. A acel eração do crescimento é um fenômeno que ocorre em crianças jovens após período de retardo do mesmo, quando a causa do déficit é removida. Há uma forma de compensação, sendo observada quando ocorre desaceleração do crescimento, denominado de reca- 
nalização e maturação. A recanalização ocorre também quando há uma aceleração do cresci mento ${ }^{22}$. Este fenômeno parece seguir alguns critérios de ajuste que provavel mente regulam a ingestão dietética de acordo com as necessi dades do organismo para reequilibrar o crescimento, embora seu mecanismo não esteja esclarecido até o presente momento. Assim, o processo de recuperação será completo ou não na dependência da severidade e da duração da desnutrição ${ }^{22}$. Walker \& Golden ${ }^{25}$, ao realizarem um estudo retrospectivo de 369 crianças desnutridas atendidas em um hospital universitário na Í ndia, com média de 12,6 meses de idade na admissão, relataram que as crianças em recuperação nutricional necessitaram alcançar um limiar de $85 \%$ do peso/i dade antes de iniciar a aceleração do crescimento linear e que esta foi mais influenciada pelo déficit inicial de estatura, embora em alguns casos tivessem iniciado a recuperação da estatura sem ter atingi do tais valores. Estes achados não foram corroborados pelos dados do presente estudo onde o ganho de estatura ocorreu simultaneamente ou às vezes prioritariamente ao de peso.

A velocidade de ganho de peso/idade não apresentou variação significante entre os quatro grupos, embora tenha sido significativamente maior quando as crianças foram divididas em pré-escolares e lactentes. Perra \& Costel $10^{26}$, em um estudo retrospectivo, avaliaram 1.038 crianças desnutridas, com idades entre 6 e 47 meses, 347 atendidas em centros de recuperação nutricional na zona rural da Guiné Bissau e 684 que não receberam atendimento, encontrando em um período de 12,6 meses de internação, incrementos médios de peso de 0,093 desvios padrão (DP) por semana, com médias de -4,52 DP na internação e de -2,76 DP na alta. Estas crianças eram consideradas recuperadas quando apresentavam $80 \%$ de adequação da mediana do padrão do NCHS para peso/idade. O ganho de peso/idade das crianças avaliadas no presente estudo apresenta um desempenho rel ativamente menor em comparação com relatos de outros centros. Entretanto comparações desse ti po são difíceis porque os centros possuem diferentes critérios de internação e em sua maioria atendem desnutridos graves enquanto as crianças deste estudo têm na sua maioria desnutrição moderada. Além disto a faixa etária das crianças varia muito, sendo que a maioria dos referidos centros atende crianças menores de 1 ano. Entre os fatores limitantes para alcançar a recuperação mais rápida das crianças, existem o número de horas de atendimento do CREN (10hs) e o número de dias por semana (5), não permitindo que o número de refeições e as quanti dades consumidas sejam suficientes para alcançar $100 \%$ das recomendacões próprias para as faixas etárias. Além disto, a escassa disponibilidade de alimentos entre as famílias das crianças atendidas, reforça a impossibilidade de complementação dos requerimentos das mesmas durante os períodos de não atendimento, como por exempl o nos fins de semana. Outro fator limitante é a capacidade gástrica da criança desnutrida que segundo Waterlow ${ }^{27}$, é cerca de $3 \%$ do seu peso. É possível ainda que esse padrão menor de recuperação em peso relacionou-se ao melhor desempenho alcançado na recuperação da estatura/idade.

Quanto à faixa etária, os dados mostraram um padrão de recuperação mais equilibrado entre peso e estatura nos pré-escolares enquanto que nos lactentes houve uma recuperação maior para estatura em relação ao peso. Segundo Prader ${ }^{22}$, a experiência clínica mostra que a recuperação é compl eta ou quase completa em crianças jovens, e o processo é semel hante ao que ocorre no esti rão da adolescência. A época de início da recuperação nutricional é determinante para que ocorra uma aceleração do crescimento, e quanto mais tarde esse processo começar, mais lenta e difícil será a recuperação linear. A média de idade no ingresso das crianças atendidas pelo CREN foi de 27 meses, quando os déficits são maiores, e mais difíceis de serem revertidos.

Quanto ao sexo, foram encontradas diferenças importantes em relação à recuperação de estatura/ idade. É provável que características hormonais específicas de acordo com o sexo possam explicar as diferenças encontradas na vel ocidade de ganho de estatura/idade, entre meninos e meninas.

\section{CONCLUSÕES}

Em resumo, os resultados do presente estudo mostram uma recuperação em termos de peso e estatura diferenciada de acordo com sexo e faixa etária e proporcional ao tempo de internação; sendo que a vel ocidade de ganho de peso foi semel hante entre os sexos porém maior nos pré-escol ares do que nos lactentes, e a velocidade de ganho de estatura foi maior entre as crianças de sexo feminino. Esses achados mostram que existem diferenças no padrão de recuperação entre lactentes e pré-escolares, e entre os sexos.

\section{SUMMARY}

\section{Pattern of nutritional recovery in malnou- rished children}

PuRPose. To assess the pattern of nutritional recovery in malnourished children. This prospective 
analysis was conducted between September 1995 and November 1996 at the Center for Recovery and Nutrition Education (CREN) in São Paulo, Brazil. Of the thirty-eight children studied, $47.4 \%$ were infants (nine girls and nine boys) and 52.6\% preschool children (twelve boys and eight girls).

METHOD. Thirty eight children were studed by velocity of $W / A$ and $H / A$ gain in standard deviation or Z score, according to weight and height median of NCHS.

RESULTS. There was a statistically significant increase in the weight-for-age $(W / A)(p=0.012)$, height-for-age $(H / A)(p=0.001)$ from the time of admission.The difference in the velocity of $W / A$ gain between infants (0.299-Z/ year) and preschool children (0.684-Z/ year) with more than six months of treatment, was found to be statistically significant ( $p=0.038)$. The difference in the velocity of $H / A$ gain for infants (0.794-Z/ year) and for preschool chidren (0.506-Z/ year), was not statistically significant ( $p=0.166)$. In regards to gender, there was not a statistically significant difference between boys and girls in the velocity of W/ A gain (0.540 and 0.524-Z/ year, respectively). The difference in velocity of $H / A$ gain was statisically significant, $0.485-Z /$ year for boys and $0.856-Z$ / year for girls $(p=0.043)$.

CONCLUSION. These findings show that there are differences in the nutritional recovery patterns between gender and age groups. [Rev Ass Med Brasil 1998; 44(4): 294-300]

KEY WORDS: Nutrition rehabilitation. Protein-energy malnutrition. Anthropometry.

\section{REFERÊ NCIAS BIBLIOGRÁFICAS}

1. Beghin Id. Nutritional Rehabilitation Centers in Latin America: a critical assessment. AmJ Clin Nutr 1970; 23: 1.412-7.

2. Beaudry-DarismeM, Latham MC. Nutritional Rehabilitation Centers - an evaluation of their performance. Environmental Child Health sept/1973; 299-332,

3. Batista Filho M, Coelho Hal. Centros de Educação e Recuperação Nutricional Primeira experiência. O Hospital 1970; 77: 13-33.

4. Pesquisa Nacional de Saúde e Nutriçaõ. Perfil decrescimento da população brasileira de 0 a 25 anos. Instituto $\mathrm{N}$ acional de Alimentação e Nutrição (INAN) Brasília, 1990.

5. Sawaya AL. Transição: desnutrição energético-proteíca e obesidade. In SAWAYA AL. Desnutrição urbana no Brasil em um período de transição. São Paulo, Cortez, 1997; 35-61.

6. Wordl Bank. Brazil: addressing nutritional problems in Brazil. Sector Report. № 1990

7. O’Donnell A, Brito S. Latin América: actions for the prevention and treatment of malnutrition. Acta Paediatr Scand Suppl 1991; 374: 168-74.

8. King KW, Fougere W, Webb RE, Berggren G, Berggren WL \&
Hilaire A. Preventive and therapeutic benefits in relation to cost: performance over 10 years of Mothercraft Centers in Haiti. AmJ Clin Nutr 1978, 31: 679-90.

9. Monckeberg GF. Desnutricion infantil: Fisiopatogía clínica tratamiento y prevención:Nuestra experiencia e contribuiçión. Santiago I NTA Universidade do Chile 1988.

10. Solymos Gmb, Sawaya AL, Ferrari AA, Vieira Mfa, Ugnebu $\mathrm{CH}$. Centro de Recuperação e Educação Nutricional: uma proposta para o combate à desnutrição energético-proteíca In SAWAYA AL. Desnutrição urbana no Brasil em um período de transição. São Paulo, Cortez, 1997; 161-93.

11. National Center For Health Statistics (NCHS). Growth Curves for Children Birth-18 years. Hyattsville Maryland 1977 DHEW Publication No PHS 78-1650 (series 11 No 165).

12. Frisancho AR. Anthropometric standards for the assesment of growth and nutritional status. Ann Arbor, University of Michigan Press 1993.

13. Lohman TJ , Roche AF, Martorell R (orgs). Anthropometric standardization referencemanual. Champaign Abridged Edition 1991.

14. WHO Physical Status: the Use e Interpretation of Anthropometry: report of a WHO expert committee. WHO technical report series: 854, 1995.

15. Martell M, Belitzky R, Gaviria J . Velocidad de crecimiento en niños nacidos pretermino y con bajo peso In: Cusminsky $M$, Moreno EM, Ojeda Ens. Crecimiento y desarrolo: hechos e tendencias. Washington DC OPAS/OMS 1988; 635 p (Publicatión Científica 510).

16. Dean AG, Dean J A, Coulombier D et al. Epi info Version 6: $A$ word processing database and statistics program for epidemiology on microcomputers. Centers for Disease Control and Prevention Atlanta Georgia USA 1994.

17. Gibson RS. Principles of nutritional assesment. New York Oxford University Press 1990.

18. Lejarraga H. La supervision del crecimiento. In: Cusminsky M, Moreno E M, Ojeda Ens. Crecimiento y desarrolo: hechos e tendencias. Washington DC OPAS/OMS 1988; 635 p (Publicatión Científica 510).

19. Tanner J M, Whitehouse RH, Takaishi M. Standards from birth to maturity for height weight height vel ocity and weight velocity: British children 1965 Part II. Arch Dis Child 1966; 41: 613-35.

20. Lampl M, Veldhuis J D, J ohnson ML. Saltation and stasis: a model of human growth Science 1992; 258: 801-2.

21. Massa E, Romana GL, Kanashiro HC et al. Una unidad de rehabilitación nutricional para el tratamientodedesnutricion infantil severa. Arch Latinoam Nutr 1980; 30: 417-31.

22. Prader A. Catch-up growth Postgrad Med J 1978; 54 (suppl 1):133-43.

23. Kirschner BS, Klich J R, Kalman SS, Defavaro MV, Rosenberg $\mathrm{IH}$. Reversal of growth retardation in Crohn's disease with therapy emphasizing oral nutritional restitution. Gastroenterology 1981; 80: 10-5.

24. Barr Dgd, Shmerling DH, Prader A. Catch-up growth in malnutrition, studied in celiac disease after institutin of gluten-free diet Pediat Res 1972; 6: 521-7.

25. Walker SP, Golden Mhn. Growth in length of children recovering from severe malnutrition Eur J Clin Nutr 1988; 42: 395-404.

26. Perra A, Costello AM de L. Efficacy of outreach nutrition rehabilitation centres in reducing mortality and improving nutritional out come of sverely malnourished children in Guinea Bissau. Eur J Clin Nutr 1995; 49: 353-9.

27. Waterlow J C. Protein-Energy Malnutrition.London, Edward Arnold, 1992 